\title{
Molecular Imaging with Macrophage CRIg-Targeting Nanobodies for Early and Preclinical Diagnosis in a Mouse Model of Rheumatoid Arthritis
}

\author{
Fang Zheng ${ }^{1,2}$, Stéphanie Put ${ }^{3}$, Luc Bouwens ${ }^{4}$, Tony Lahoutte ${ }^{5,6}$, Patrick Matthys ${ }^{3}$, Serge Muyldermans ${ }^{1,7}$, Patrick De \\ Baetselier $^{1,2}$, Nick Devoogdt ${ }^{* 5}$, Geert Raes*1,2, and Steve Schoonooghe*1,2 \\ ${ }^{1}$ Laboratory of Cellular and Molecular Immunology, Vrije Universiteit Brussel, Brussels, Belgium; ${ }^{2}$ VIB Laboratory of Myeloid Cell \\ Immunology, Brussels, Belgium; ${ }^{3}$ Laboratory of Immunobiology, Rega Institute, Katholieke Universiteit Leuven, Leuven, Belgium; \\ ${ }^{4}$ Cell Differentiation Unit, Diabetes Research Centre, Vrije Universiteit Brussel, Brussels, Belgium; ${ }^{5}$ In Vivo Cellular and Molecular \\ Imaging Center, Vrije Universiteit Brussel, Brussels, Belgium; ${ }^{6}$ Department of Nuclear Medicine, UZ Brussel, Vrije Universtiteit \\ Brussel, Brussels, Belgium; and ${ }^{7}$ VIB Department of Structural Biology, Vrije Universiteit Brussel, Brussels, Belgium
}

\begin{abstract}
An accurate and noninvasive tracer able to detect molecular events underlying the development of rheumatoid arthritis (RA) would be useful for RA diagnosis and drug efficacy assessment. A complement receptor of the lg superfamily (CRlg) is expressed on synovial macrophages of RA patients, making it an interesting target for molecular imaging of RA. We aim to develop a radiotracer for the visualization of CRIg in a mouse model for RA using radiolabeled single-domain variable antibody VHH fragments (Nanobodies). Methods: Quantitative polymerase chain reaction was used to locate CRIg expression in mice with collagen-induced arthritis (CIA). A Nanobody, NbV4m119, was generated to specifically target CRIg. Flow cytometry, phosphorimaging, and confocal microscopy were used to confirm NbVm119 binding to CRlg-positive cells. SPECT (SPECT/CT) was used to image arthritic lesions in the inflamed paws of 29 mice using ${ }^{99 m T c-}$ NbV4m119 Nanobody. Results: CRlg is constitutively expressed in the liver and was found to be upregulated in synovial tissues of CIA

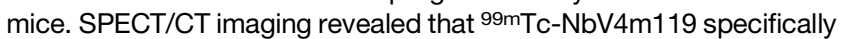
targeted CRIg-positive liver macrophages in naïve wild-type but not in $\mathrm{CRIg}^{-/-}$(CRlg knockout) mice. In ClA mice, ${ }^{99 m T c-N b V 4 m 119 ~ a c-~}$ cumulation in arthritic lesions increased according to the severity of the inflammation. In the knees of mice with CIA, ${ }^{99 m T c-N b V 4 m 119 ~}$ was found to accumulate even before the onset of macroscopic clinical symptoms. Conclusion: SPECT/CT imaging with 99mTcNbV4m119 visualizes joint inflammation in CIA. Furthermore, imaging could predict which mice will develop clinical symptoms during CIA. Consequently, imaging of joint inflammation with CRIg-specific Nanobodies offers perspectives for clinical applications in RA patients.
\end{abstract}

Key Words: complement receptor of the Ig superfamily (CRIg, VSIG4, or Z39lg); collagen-induced arthritis (CIA); SPECT/MCT; Nanobody; macrophage

J Nucl Med 2014; 55:824-829

DOI: $10.2967 /$ jnumed.113.130617

Received Aug. 7, 2013; revision accepted Jan. 1, 2014.

For correspondence or reprints contact: Steve Schoonooghe, Laboratory of Cellular and Molecular Immunology, VIB Laboratory of Myeloid Cell Immunology, Vrije Universiteit Brussel, Pleinlaan 2, 1050 Brussels, Belgium.

E-mail: sschoono@vub.ac.be

${ }^{*}$ Contributed equally to this work.

Published online Mar. 31, 2014.

COPYRIGHT (C 2014 by the Society of Nuclear Medicine and Molecular Imaging, Inc.
D comes hyperplastic because of local proliferation of fibroblastlike synoviocytes and increased infiltration of various immune cells $(1,2)$. Notably, mononuclear cell infiltration of the intimal lining leads to erosion of cartilage and subchondral bone $(3,4)$. Additionally, macrophages accumulate earlier and continuously in the intimal layer, numbering up to $50 \%$ of the total cells $(5,6)$. Indeed, the presence of specific macrophage populations can be used to monitor inflammation in RA. For instance, more cells expressing the complement receptor of the Ig superfamily (CRIg, aka VSIG4, Z39Ig) were found in the synovial lining layer of RA patients than in other arthritic pathologies or normal synovium (7). In humans, CRIg has a pronounced expression on Kupffer cells and is found to a certain extent on resident tissue macrophage subsets in various tissues, including normal synovium $(8,9)$. However, both messenger RNA (mRNA) analysis and immunohistochemical staining on synovium of clinical RA patients have revealed that CRIg is more abundant in RA synovial tissue and is coexpressed with genes from activated macrophages in both the lining and the sublining layers of the synovium $(7,9,10)$. Consequently, CRIg seems to be a promising marker for specific molecular imaging of RA, allowing noninvasive quantification of joint inflammation in vivo.

SPECT/micro-CT and other forms of scintigraphy with radioactive labels conjugated to cytokines, antibodies, or antibody fragments allow monitoring of disease progression using physiologic parameters and the simultaneous evaluation of specific marker expression in every inflammatory lesion $(11,12)$. Nanobodies are small $(15 \mathrm{kDa})$ single-domain derivatives from camelid heavy-chain antibodies that can bind their target quickly and specifically. Concurrently, unbound Nanobodies are rapidly eliminated from the body, which increases the contrast between marker-specific and background signals in molecular imaging (13). Their good solubility and high intrinsic thermostability allows for straightforward radiolabeling $(13,14)$. Nanobody-derived probes have already been shown to generate high target-to-background and specific images within $1-3 \mathrm{~h}$ of tracer injection in several mouse tumor and inflammation models (15-18). Recently, we demonstrated by mouse macrophage mannose receptor (MMR)-specific Nanobody imaging that macrophages are located in the naive liver, spleen, lymph nodes, 
bone marrow, and inflamed paws of mice with collagen-induced arthritis (CIA) $(16,19)$.

In the present study, searching for a marker with a more restricted expression, we investigated the in vivo biodistribution of CRIg in naive mice and in mice with CIA, examining joint tissues and specifically focusing on synovial macrophages. We developed and characterized CRIg-specific Nanobodies and evaluated Nanobody-based radiolabeled tracers for preclinical imaging of arthritic lesions. Finally, we gauged whether CRIg expression levels could predict joint inflammation in animals before the appearance of clinical symptoms.

\section{MATERIALS AND METHODS}

\section{Mice}

Male C57BL/6 and DBA/1 mice (age, 8 wk) were purchased from Janvier Sas. CRIg-deficient mice (C57BL/6 background) were generously provided by Genentech. The experiments were approved by the local ethics committee. CIA was induced as described before (19). Briefly, chicken collagen type II ( $2 \mathrm{mg} / \mathrm{mL})$ (Sigma-Aldrich) in $0.1 \mathrm{M}$ acetic acid was emulsified in complete Freund adjuvant (Difco Laboratories) with added heat-killed Mycobacterium butyricum $(0.75 \mathrm{mg} / \mathrm{mL}$; Difco Laboratories). Mice were sensitized with a subcutaneous injection at the base of the tail with a 100- $\mu \mathrm{L}$ emulsion. A boost injection with chicken collagen type II $(2 \mathrm{mg} / \mathrm{mL})$ in incomplete Freund adjuvant was performed at day 21. Each limb was scored for severity of arthritis as follows: 0 , normal; 1 , redness/swelling of one joint; 2 , redness/swelling of more than one joint; 3, swelling of entire paw; and 4, ankylosis or deformity (20).

\section{Quantitative Real-Time Polymerase Chain Reaction}

mRNA levels were determined as described previously (20). A brief description is given in the supplemental information (available at http://jnm.snmjournals.org).

\section{Antibodies and Nanobodies}

Anti-CD45.2 (Cl:104)/fluorescein isothiocyanate (FITC), anti-CD11b (M1/70)/PE-Cy7, and anti-F4/80(CI:BM8)/allophycocyanin (APC)-eFluor780 antibodies were purchased from eBioscience. Anti-CRIg monoclonal antibody was generously provided by Genentech; the corresponding isotype control antibody was purchased from R\&D Systems. Nanobodies were generated and produced as described previously (21) and as detailed in the supplemental information. Nanobodies against the $\beta$-lactamase BCII enzyme of Bacillus cereus (BCII10) were used as a negative control throughout the study. Nanobodies were labeled using the Alexafluor647 protein-labeling kit (Invitrogen) according to the manufacturer's instructions. For immunofluorescence microscopy, Nanobodies were subcloned into the pMECS vector, which adds a hemaglutinin tag to the VHH. Anti-CD68/biotin was purchased from AbD Serotec and anti-hemaglutinin-tag-AlexaFluor488 was purchased from Invitrogen. Inflamed knee synovia were embedded in Tissue-Tek OCT (Sakura) and frozen in liquid nitrogen. Cryostat sections $(5 \mu \mathrm{m})$ were fixed in 3\% paraformaldehyde (pH 7.4) and incubated in $1 \%$ phosphatebuffered saline/blocking reagent (Roche) containing detection antibodies before imaging on a multiphoton laser-scanning microscope (LSM710 and ConfoCor 3 system; Zeiss). The detailed procedure is described in the supplemental information.

\section{9mTc Nanobody Labeling, Pinhole SPECT/micro-CT \\ Analysis, and Phosphorimaging \\ Nanobodies were labeled with ${ }^{99 \mathrm{~m}} \mathrm{Tc}$ via their polyhistidine (his) tag as described before $(21,22)$. C57BL/6J mice or DBA/1 mice were injected intravenously with $80-100 \mu \mathrm{L}$ of ${ }^{99 \mathrm{~m}} \mathrm{Tc}$ Nanobodies. The ${ }^{99 \mathrm{~m}} \mathrm{Tc}$ Nano- body activity injected per mouse was $52.80 \pm 19.63 \mathrm{MBq}(1.427 \pm$ $0.5306 \mathrm{mCi})(n=90$, mean $\pm \mathrm{SEM})$. SPECT/micro-CT was performed as described previously (20). A brief description is given in the supple-}

mental information. Immediately after imaging, mice were sacrificed, and tissue sections were phosphorimaged as described in the supplemental information.

\section{Statistical Analysis}

Statistical analyses were conducted using the Student $t$ test and 1-way ANOVA assuming unequal variances. Prism 5.0 (GraphPad Software) was used for statistical analyses and graph creation. $P$ values of 0.05 or less were considered significant.

\section{RESULTS}

\section{Expression of CRIg}

Direct proof of CRIg expression in mouse synovial cells is so far lacking. Therefore, CRIg mRNA levels were investigated in CIA mice to determine whether this marker is widely expressed in the body or whether it is limited to specific tissues during inflammatory conditions. When CRIg mRNA levels were determined for multiple tissues of CIA mice, a high level of expression was found in the liver and low or undetectable quantities were measured in the bone marrow, lymph nodes, spleen, and lung. CRIg expression was substantially higher for the synovium and synovial fluid than for other organs (Supplemental Fig. 1).

\section{Tracking CRIg Expression In Vivo in Naive and CIA Mice Using CRIg-Specific Nanobodies}

CRIg-specific Nanobodies were prepared from lymphocytes isolated from an alpaca immunized with the extracellular domain of mouse CRIg recombinant protein, as described before for other Nanobodies (20). The lead candidate, NbV4m119, has an affinity of $0.9 \mathrm{nmol} / \mathrm{L}$ for recombinant $\mathrm{mCRIg}$, and flow cytometry analysis confirmed that it recognizes native mouse CRIg expressed on liver

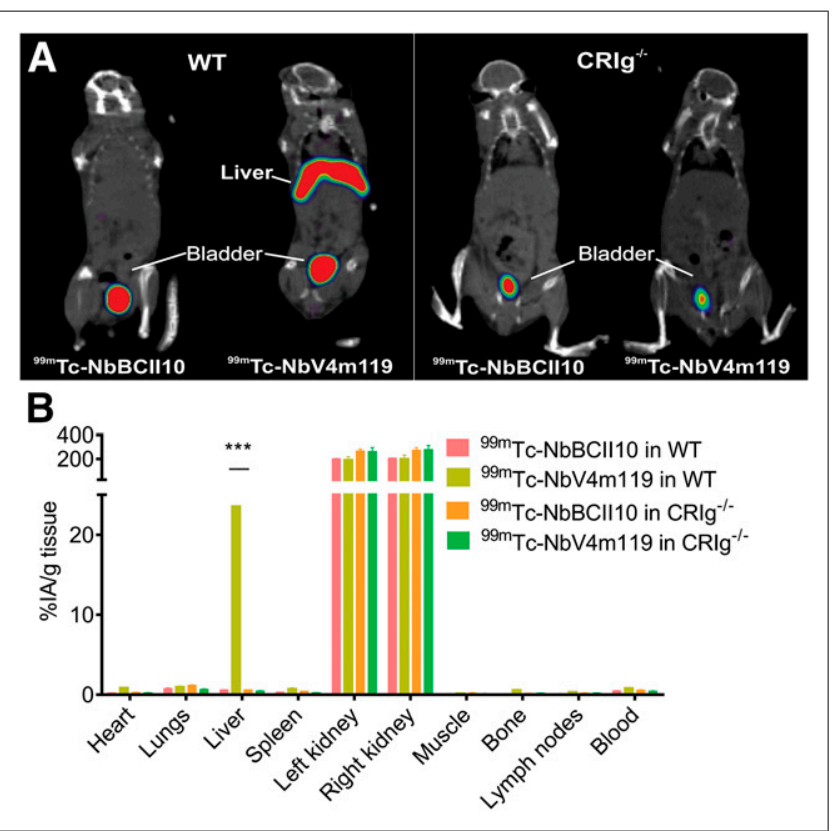

FIGURE 1. (A) Coronal views of SPECT/micro-CT imaging performed on naive wild-type and $\mathrm{CRIg}^{-/-}$mice $1 \mathrm{~h}$ after intravenous injection of 99mTc-labeled NbBCII10 and NbV4m119. Representative images for 3 mice per group are shown using National Institutes of Health color scale and are scaled to maximum in whole image. (B) Radioactive content of each organ was measured in y counter and expressed as percentage injected activity per gram of tissue $(\% \mathrm{IA} / \mathrm{g})(n=3$ mean \pm SEM, ${ }^{\star \star \star} P<0.001, \mathrm{NS}=$ not significant vs. ${ }^{99 m T c-N b B c l l 10) .} \mathrm{WT}=$ wild-type. 


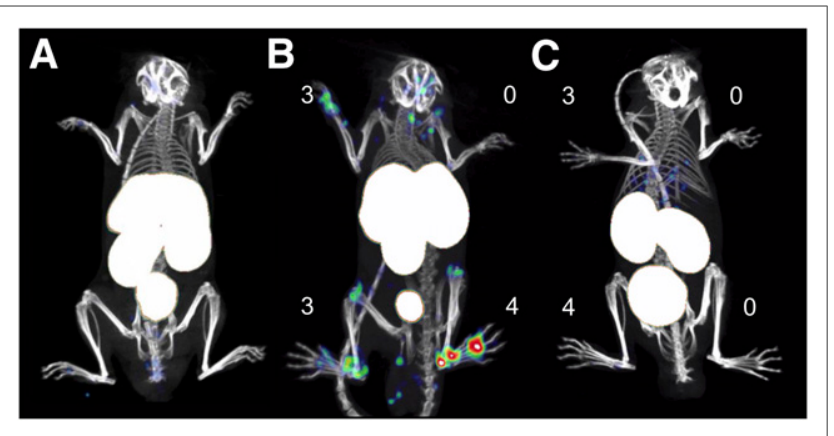

FIGURE 2. In vivo SPECT/CT imaging with anti-CRIg Nanobody tracer visualizes CRIg expression in arthritic joints of CIA mice. Representative SPECT/micro-CT image of CIA mice, $3 \mathrm{~h}$ after injection with $99 \mathrm{mTC}-$ labeled Nanobodies V4m119 or control BCIl10. DBA/1 naive mice (A) did

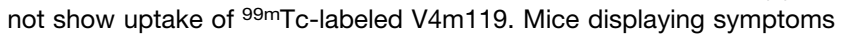
of arthritis showed specific uptake of ${ }^{99 \mathrm{mTC}} \mathrm{T}$-labeled V4m119 in inflamed joints in correlation with clinical scores (B) but showed no uptake of 99mTc-labeled BCII10 control Nanobody (C). Clinical scores are indicated next to each joint. Representative images of 3 mice per group are shown using National Institutes of Health color scale and are scaled to maximum in whole image.

macrophages ex vivo (Supplemental Fig. 2). To confirm the specificity of NbV4m119, whole-mouse SPECT/CT imaging was performed on naive $\mathrm{CRIg}^{-1-}$ (CRIg knockout) and C57BL/6J counterparts injected with $99 \mathrm{~m}$ Tc-labeled Nanobodies. In concordance with the tissue mRNA expression studies, ${ }^{99 m}$ Tc-NbV4m119 Nanobody uptake in the liver of wild-type animals was higher than the nontargeting control ${ }^{99 \mathrm{~m}} \mathrm{Tc}-\mathrm{NbBCII} 10$, a Nanobody binding a bacterial enzyme $(P<0.05)$ (Fig. 1A). Additionally, only low levels of CRIg $(<2$ percentage injected activity per gram of tissue) could be detected in all other investigated tissues, including the joints. In the SPECT/CT images, no specific uptake of ${ }^{99 \mathrm{~m} T c-N b V 4 \mathrm{~m} 119}$ could be visualized in the liver or other organs of $\mathrm{CRIg}^{-/-}$mice, verifying the in vivo targeting specificity of the Nanobody (Fig. 1A). Further confirmation of this was provided by dissection of the different organs and measurement of the residual radioactivity in a $\gamma$ counter (Fig. 1B). ${ }^{99 \mathrm{~m}} \mathrm{Tc}-\mathrm{NbV} 4 \mathrm{~m} 119$ and the control ${ }^{99 \mathrm{~m}} \mathrm{Tc}-$ NbBCII10 exhibited high kidney uptake and high activities in the bladder for both Nanobodies in $\mathrm{CRIg}^{-1-}$ and wild-type mice. These signals are associated with the fast filtration of unbound Nanobody from the bloodstream and are therefore not attributable

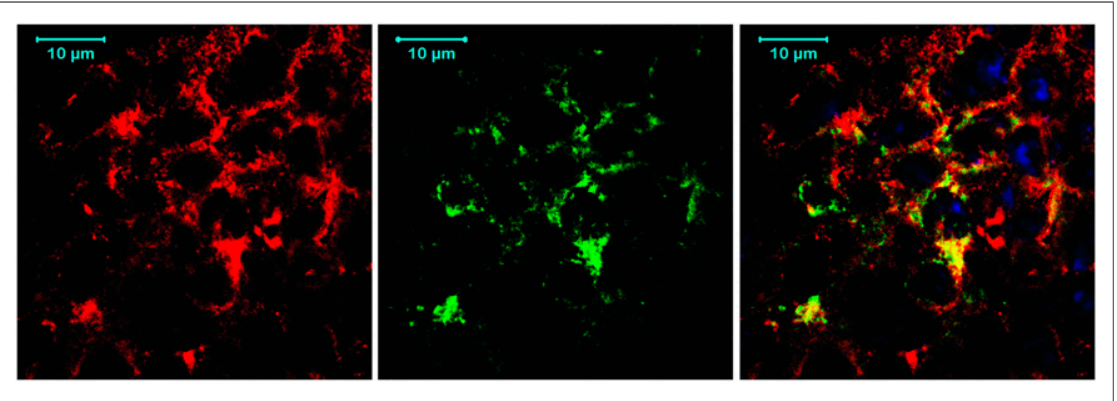

FIGURE 3. Immunofluorescence staining for CRIg and CD68 in synovium of inflamed knee $(n=3)$. Formalin-preserved cryosections of inflamed knee synovium were stained with anti-CD68-biotin and Alexafluor568-streptavidin (left) and with hemaglutinin-tagged NbV4m119 and Alexafluor488conjugated antihemaglutinin antibody (middle). Merged image (right) shows colocalization of CRIg expression with subset of CD68-positive macrophages. 4',6-diamidino-2-phenylindole (DAPI) (blue) was used as nuclear stain. to differences in specific Nanobody accumulation. As a result, NbV4m119 is able to specifically measure CRIg levels in vivo and was therefore used as a CRIg probe in the CIA model in

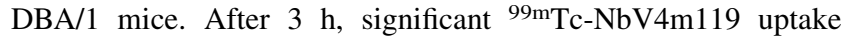
was observed only in the kidneys, bladder, and liver of naive DBA/1 mice (Fig. 2A). The CIA mice were scored for arthritis severity and subsequently injected with ${ }^{99 \mathrm{~m}} \mathrm{Tc}-\mathrm{NbV} 4 \mathrm{~m} 119$ or ${ }^{99 \mathrm{~m} T c-N b B C I I 10 .}{ }^{99 \mathrm{~m}} \mathrm{Tc}-\mathrm{NbV} 4 \mathrm{~m} 119$ accumulated specifically in the inflamed joints of arthritic mice (Fig. 2B; Supplemental Video 1). No significant joint uptake was detected in ${ }^{99 \mathrm{~m}} \mathrm{Tc}-\mathrm{NbBCII} 10$ injected arthritic mice (Fig. 2C; Supplemental Video 2).

\section{Localization of CRIg-Positive Cells}

For a more precise localization of the CRIg signal observed in CIA mice, inflamed joints of ${ }^{99 m} \mathrm{Tc}-\mathrm{NbV} 4 \mathrm{~m} 119$-challenged animals were dissected to quantify the radioactivity in the dissected parts. After removing the synovium from the knees and ankles of the CIA mice, the uptake of ${ }^{99 \mathrm{~m} T c-N b V 4 m 119}$ in the hind limb decreased significantly $(P<0.05)$ (Supplemental Fig. 3), indicat-

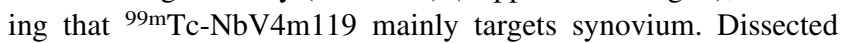
samples exposed to radiosensitive screens confirmed that ${ }^{99 \mathrm{~m}} \mathrm{Tc}-$ NbV4m119 accumulates mainly in synovium from arthritic knees and ankles ( $P<0.05$ vs. NbBCII10, Supplemental Fig. 4). Immunofluorescence analysis of inflamed knee synovium sections revealed that $\mathrm{NbV4m119}$ targets a subset of CD68-positive macrophages, presumably macrophagelike (type A) synoviocytes (Fig. 3).

\section{9mTc-NbV4m119 Uptake in Relation to Clinical Arthritic Scores and Disease Severity}

Among collagen type II-immunized mice, some remained asymptomatic (no macroscopic evidence of arthritis in any paw) and others became symptomatic. In the latter, individual paws were assigned clinical scores ( $0-4$ for severe inflammation). ${ }^{99 \mathrm{~m} T c-N b V 4 m 119}$ uptake was readily visualized in arthritic lesions from the symptomatic CIA mice and correlated with the clinical scoring (Fig. 4A). However, in contrast to the clinical assessment, the uptake of ${ }^{99 \mathrm{~m} T c-N b V 4 \mathrm{~m} 119}$ not only was associated with increased disease severity, but also yielded information on inflammation intensity in individual joints, including those not considered in the clinical scoring system, such as the knees (Supplemental Video 3). Knees from symptomatic mice accumulated more ${ }^{99 \mathrm{~m}} \mathrm{Tc}-\mathrm{NbV} 4 \mathrm{~m} 119$ than knees from asymptomatic mice $(P<0.05)$ (Fig. 4B). This enhanced uptake of ${ }^{99 \mathrm{~m}} \mathrm{Tc}-$ $\mathrm{NbV} 4 \mathrm{~m} 119$ in knees from symptomatic versus asymptomatic mice was detected irrespective of the clinical score of the limb and even occurred in a subset of knees from limbs for which no clinical symptoms could yet be detected macroscopically (score 0 paws of symptomatic mice). In the metatarsal joints, a significant increase in 99m Tc-NbV4m119 accumulation was detected only in limbs of score 2 and score $3-4(P<$ $0.05)$ as compared with joints of asymptomatic mice (Fig. 4C). Finally, ankles only accumulated significantly more ${ }^{99 \mathrm{~m} T c-N b V 4 \mathrm{~m} 119}$ versus asymptomatic mice in limbs with scores 3-4 $(P<0.05)$ (Fig. 4D).

\section{In Vivo NbV4m119 Accumulation Before Onset of Arthritis}

The observation that ${ }^{99 \mathrm{~m}} \mathrm{Tc}-\mathrm{NbV} 4 \mathrm{~m} 119$ signals were elevated in a subset of joints 


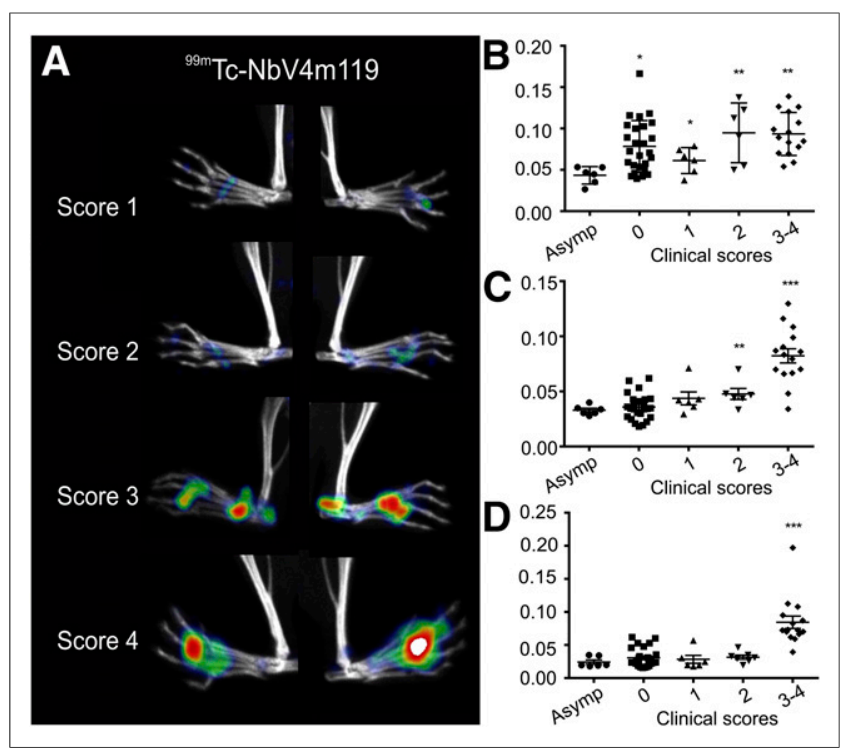

FIGURE 4. SPECT/micro-CT image quantification of joints with various clinical arthritic scores at $3 \mathrm{~h}$ after injection of ${ }^{99 \mathrm{mTc}} \mathrm{T}-\mathrm{V} 4 \mathrm{~m} 119$. Mice immunized with collagen type $\|$ in complete Freund adjuvant were sorted into asymptomatic (total clinical scores of all limbs, 0) and symptomatic mice. SPECT/micro-CT imaging of individual limbs of symptomatic mice, grouped according to their clinical scores $(A)$, revealed that 99mTc-V4m119 accumulation intensifies with increasing clinical scores. Representative images (of 58) of hind limbs are shown using National Institutes of Health color scale and are scaled to maximum in whole image. Radioactive signals in regions of interest were quantified for knees (B), metatarsal joints (C), and ankles (D) of $29 \mathrm{ClA}$ mice as percentage injected activity (mean \pm SEM). ${ }^{*} P<0.05$, ${ }^{* *} P<0.01$, ${ }^{\star \star \star} P<0.001$ vs. joints from asymptomatic mice (Asymp).

without clinically visible symptoms inspired us to investigate the potential of this tracer to predict the occurrence of CIA before clinical onset of the disease. Therefore, SPECT/CT imaging was performed with this Nanobody on day 23 after immunization. Signals were quantified in the joints of the front paws, knees, metatarsal joints, and ankles. Interestingly, the mean level of radioactivity in the knees of CIA mice that developed symptoms after the day of imaging was significantly higher than asymptomatic mice, which did not show symptoms until day $32(P<0.05)$ (Fig. 5A). Other inflamed joints did not show this early signal of ${ }^{99 \mathrm{~m}} \mathrm{Tc}-\mathrm{NbV} 4 \mathrm{~m} 119$ before symptoms arose. Hot spots of ${ }^{99 \mathrm{~m}} \mathrm{Tc}-\mathrm{NbV} 4 \mathrm{~m} 119$ were observed in SPECT imaging of knee joints both in symptomatic mice (Fig. 5B) and in mice that did not have any macroscopic signs of arthritis yet (Fig. 5C; Supplemental Video 4). In contrast, mice that remained asymptomatic did not show any focal uptake of 99mTc-NbV4m119 (Fig. 5D).

\section{DISCUSSION}

Modern imaging techniques are becoming increasingly important in assessing the course of inflammatory ailments and in permitting treatment follow-up for these diseases. Although contrast-enhanced MR imaging and ultrasonography are used to image synovial fluid effusion, neoangiogenesis, and vasodilation, they are still generally limited to delineating lesions at the anatomic level (23). Molecular imaging with SPECT or PET can greatly complement these techniques by providing information on the presence of specific molecules and cell types in the joints at any one time. Several approaches have already been explored for molecular imaging of
RA, including MMPSense or ProSense (24,25), small-molecule probes targeting the folate receptor (26), and antibody-based methods directed against E-Selectin or ICAM-1 $(12,27)$. The key to the success of these approaches is the marker being targeted as well as the specificity and sensitivity of the tracer. In arthritis, the cells responsible for most of the chronic inflammation and progressive joint destruction are located in the synovial membrane, making them a good target for tracking arthritis. We have previously described successful targeting of arthritic joints in CIA mice using an antiMMR Nanobody. However, in addition to the Nanobody accumulation observed in diseased joints, the Nanobody was also found in the liver, spleen, heart, lymph nodes, and bone marrow (20). Although the MMR Nanobody targets macrophages present in the joints of CIA mice, the MMR signal in the bone marrow could be problematic for the accurate imaging of some inflamed joints (16). Macrophages are attractive targets for imaging because they seem to have a pivotal role in maintaining the chronic inflammation state in arthritis (9). Therefore, a marker largely restricted to these cells could further improve the accuracy of SPECT imaging in arthritis. In this respect, CRIg seems to be a suitable target, for it has been shown to be expressed on resident tissue macrophages in normal human synovium and on the corresponding macrophages in RA patients' synovial lining layer (7). In the present study, we aimed to investigate specifically the expression of CRIg in CIA, a mouse model that mimics well the immunologic state in human RA (19). In general, CRIg was hardly detectable in bone marrow, lymph nodes, and spleen, corresponding to previous observations $(7,8,28)$.

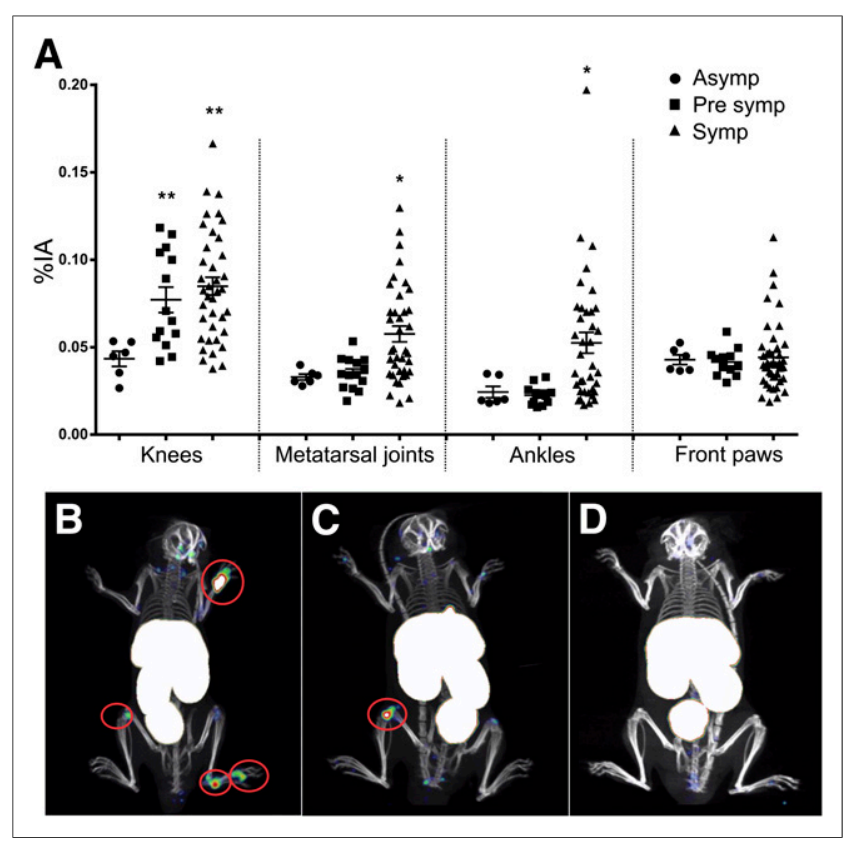

FIGURE 5. Quantification of $99 \mathrm{mTc}-\mathrm{V} 4 \mathrm{~m} 119$ accumulation in joints of $\mathrm{CIA}$ mice before onset of arthritis symptoms. CIA mice at day 23 after immunization were imaged and subsequently sorted in 3 groups. (A)

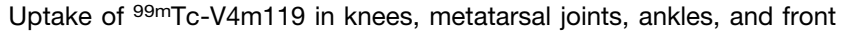
paws of CIA mice $23 \mathrm{~d}$ after immunization $(n=29)$. Percentage injected activity was quantified for each area of interest. ${ }^{*} P<0.05,{ }^{\star *} P<0.01$, vs. asymptomatic mice. Representative SPECT/micro-CT images of symptomatic (B), presymptomatic (C), and asymptomatic (D) mice. Asymp $=$ asymptomatic, mice free of symptoms until day 32 (experiment end); Pre symp = presymptomatic, mice with arthritis symptoms after day 23; Symp = symptomatic, mice with arthritis symptoms on day 23. $\% \mid \mathrm{A}=$ percentage injected activity. 
High levels of CRIg were found on CD11 b intermediate $^{2} 4 / 80^{+}$Kupffer cells in the liver. In CIA mice, CRIg was present in the inflamed synovium on cells expressing the pan-macrophage marker CD68 (29), agreeing with previous studies on synovial tissue from RA patients $(7,9)$ and appearing to confirm its value for in vivo imaging of joint inflammation. We have chosen Nanobodies as tools for this study because these small binding moieties suffer less from high background signals, as compared with complete antibodies, and penetrate deep into tissues whereas unbound molecules are cleared rapidly from the body, so high-contrast images can be acquired in a matter of hours after injecting the probe. In contrast, standard inflammation imaging probes such as ${ }^{18} \mathrm{~F}-\mathrm{FDG}$ suffer from higher background signals than these antigen-specific probes. The signals observed with ${ }^{18} \mathrm{~F}$-FDG are generally higher in inflamed joints than with Nanobodies; however, many of these signals are aspecific. Furthermore, Nanobodies do not bind to $\mathrm{Fc}$ receptors and have not been reported to induce strong neutralizing immune responses. They can be produced in high quantities using bacterial expression systems, and their thermostability makes radiolabeling more straightforward than for many other proteins. Comparing the biodistribution of ${ }^{99 m} \mathrm{Tc}-\mathrm{CRIg}-$ specific Nanobodies in naive mice with that of ${ }^{99} \mathrm{~m}$ Tc-labeled control BCII10 Nanobodies revealed a strong accumulation of ${ }^{99 \mathrm{~m}} \mathrm{Tc}-\mathrm{V} 4 \mathrm{~m} 119$ in the liver but not in any other organ, confirming in vivo the immunohistochemical results reported by Vogt et al. $(8,9,28)$. The CRIg specificity of the signal produced by the Nanobody was further confirmed by a total absence of Nanobody accumulation in the livers of $\mathrm{CRIg}^{-1-}$ mice. The signals associated with kidneys and bladders were similar for wild-type and $\mathrm{CRIg}^{-1-}$ mice, indicating that they reflect the physiologic process of reuptake after clearance and not an in situ CRIg-specific binding. Because the presence of a His-tag significantly affects kidney retention, the kidney uptake seen in this preclinical work reflects the uptake of a clinical-grade probe (30). Several ways exist to limit aspecific kidney retention such as using other labeling chemistries or radionuclides and adding substances that limit renal uptake (31). Taken together, these results verify that in naive nonimmunized mice, the liver is indeed the only main organ containing cells expressing substantial amounts of CRIg. When injected in mice with active CIA, a ${ }^{99 \mathrm{~m}} \mathrm{Tc}-\mathrm{V} 4 \mathrm{~m} 119$ Nanobody-specific signal was also observed in the inflamed joints. Although the absolute quantity of ${ }^{99 \mathrm{~m}} \mathrm{Tc}-\mathrm{V} 4 \mathrm{~m} 119$ Nanobody in the inflamed joints is low, an excellent contrast with the background was achieved. The Nanobody accumulation in the joints was also correlated to the severity of the disease as judged by the macroscopic arthritis scoring system. However, uptake of the CRIg Nanobody in different types of joints reveals additional variability that goes beyond the limitations of this scoring system, giving information on inflammation intensity in individual joints, including those not considered during scoring. Interestingly, in the early stages of the CIA ${ }^{99} \mathrm{~m}$ TcNbV4m119 accumulation was also observed in the joints, primarily knees, of mice that had yet to develop any symptoms of arthritis but that became symptomatic later on. The knees of mice that remained free of arthritis did not show such accumulation of CRIg-specific Nanobody. Such a difference was not observed for the control Nanobody nor in our previous results with an anti-MMR Nanobody (20), indicating that the increased presence of CRIg in a joint can provide an indication of incipient inflammation in as-of-yet asymptomatic paws. Furthermore, the flexibility of conjugation and labeling of Nanobodies allows other possibilities of imaging using CRIg-specific Nanobodies, such as PET scanning, near-infrared fluorescence imaging
$(26,27,32)$, or coupling to microbubbles for ultrasound-based imaging (33). Moreover, Nanobodies have low toxicity and low immunogenicity, with a clinical study using Nanobody PET tracers currently running. Additionally, CRIg is present in the joints of human RA patients. Thus, translation of a CRIg-specific Nanobody to the clinic for imaging of human RA is a realistic possibility and could be a boon for the early diagnosis of human patients.

\section{CONCLUSION}

Inducing CIA increases the amount of CRIg expressed on macrophages in inflamed mouse joints. In naive animals, only the liver holds significant amounts of CRIg-positive cells. SPECT imaging with ${ }^{99 m}$ Tc-CRIg-specific Nanobodies is a fast and accurate technique to visualize and quantify joint inflammation in an animal model of RA. Furthermore, the technique seems sufficiently sensitive to detect early signs of inflammation even before the manifestation of anatomically visible signs of the disease. The detection of early signs of inflammation would greatly benefit the diagnosis, grading, and therapy monitoring of RA.

\section{DISCLOSURE}

The costs of publication of this article were defrayed in part by the payment of page charges. Therefore, and solely to indicate this fact, this article is hereby marked "advertisement" in accordance with 18 USC section 1734. Support grants were received from the government agency for Innovation by Science and Technology, the interuniversity attraction poles, and the Concerted Research Actions of the Regional Government of Flanders. Tony Lahoutte is senior clinical investigator for the Research Foundation Flanders and the National Fund for Scientific Research-Flanders. Fang Zheng has a scholarship from the Top-Notch Student Scholarship Fund of China Scholarship Council. No other potential conflict of interest relevant to this article was reported.

\section{ACKNOWLEDGMENTS}

We thank Cindy Peleman, Eddy Himpe, Dr. Benoit Stijlemans, and Yannick Morias for technical assistance and Dr. Alain Beschin for useful discussions and contributions to identifying CRIg as a target marker on myeloid cells.

\section{REFERENCES}

1. McInnes IB, Schett G. The pathogenesis of rheumatoid arthritis. $N$ Engl J Med. 2011;365:2205-2219.

2. Veale D, Yanni G, Rogers S, Barnes L, Bresnihan B, Fitzgerald O. Reduced synovial membrane macrophage numbers, ELAM-1 expression, and lining layer hyperplasia in psoriatic arthritis as compared with rheumatoid arthritis. Arthritis Rheum. 1993;36:893-900.

3. Fonseca JE, Edwards JC, Blades S, Goulding NJ. Macrophage subpopulations in rheumatoid synovium: reduced CD163 expression in CD4+ T lymphocyte-rich microenvironments. Arthritis Rheum. 2002;46:1210-1216.

4. Ospelt C, Gay S. The role of resident synovial cells in destructive arthritis. Best Pract Res Clin Rheumatol. 2008;22:239-252.

5. Smeets TJ, Kraan MC, van Loon ME, Tak PP. Tumor necrosis factor alpha blockade reduces the synovial cell infiltrate early after initiation of treatment, but apparently not by induction of apoptosis in synovial tissue. Arthritis Rheum. 2003;48:2155-2162.

6. Szekanecz Z, Koch AE. Macrophages and their products in rheumatoid arthritis. Curr Opin Rheumatol. 2007;19:289-295.

7. Tanaka M, Nagai T, Tsuneyoshi Y, et al. Expansion of a unique macrophage subset in rheumatoid arthritis synovial lining layer. Clin Exp Immunol. 2008;154:38-47. 
8. Helmy KY, Katschke KJ Jr, Gorgani NN, et al. CRIg: a macrophage complement receptor required for phagocytosis of circulating pathogens. Cell. 2006;124:915-927.

9. Lee MY, Kim WJ, Kang YJ, et al. Z39Ig is expressed on macrophages and may mediate inflammatory reactions in arthritis and atherosclerosis. J Leukoc Biol. 2006;80:922-928.

10. Walker MG. Z39Ig is co-expressed with activated macrophage genes. Biochim Biophys Acta. 2002;1574:387-390.

11. Irmler IM, Opfermann T, Gebhardt $P$, et al. In vivo molecular imaging of experimental joint inflammation by combined ${ }^{18} \mathrm{~F}$-FDG positron emission tomography and computed tomography. Arthritis Res Ther. 2010;12:R203.

12. Lee SI, Lee SY, Yoon KH, et al. Molecular MR imaging for visualizing ICAM-1 expression in the inflamed synovium of collagen-induced arthritic mice. Korean J Radiol. 2009;10:472-480.

13. Wesolowski J, Alzogaray V, Reyelt J, et al. Single domain antibodies: promising experimental and therapeutic tools in infection and immunity. Med Microbiol Immunol (Berl). 2009;198:157-174.

14. Vaneycken I, D'huyvetter M, Hernot S, et al. Immuno-imaging using nanobodies. Curr Opin Biotechnol. 2011;22:877-881.

15. Vaneycken I, Devoogdt N, Van Gassen N, et al. Preclinical screening of antiHER2 nanobodies for molecular imaging of breast cancer. FASEB J. 2011;25: 2433-2446.

16. Movahedi K, Schoonooghe S, Laoui D, et al. Nanobody-based targeting of the macrophage mannose receptor for effective in vivo imaging of tumor-associated macrophages. Cancer Res. 2012;72:4165-4177.

17. Broisat A, Hernot S, Toczek J, et al. Nanobodies targeting mouse/human VCAM1 for the nuclear imaging of atherosclerotic lesions. Circ Res. 2012;110:927-937.

18. Vosjan MJ, Perk LR, Roovers RC, et al. Facile labelling of an anti-epidermal growth factor receptor Nanobody with ${ }^{68} \mathrm{Ga}$ via a novel bifunctional desferal chelate for immuno-PET. Eur J Nucl Med Mol Imaging. 2011;38:753-763.

19. Williams RO. Collagen-induced arthritis in mice. Methods Mol Med. 2007;136: 191-199.

20. Put S, Schoonooghe S, Devoogdt N, et al. SPECT imaging of joint inflammation with Nanobodies targeting the macrophage mannose receptor in a mouse model for rheumatoid arthritis. J Nucl Med. 2013;54:807-814.

21. De Groeve K, Deschacht N, De Koninck C, et al. Nanobodies as tools for in vivo imaging of specific immune cell types. J Nucl Med. 2010;51:782-789.
22. Cortez-Retamozo V, Lahoutte T, Caveliers V, et al. ${ }^{99 \mathrm{~m} T c-l a b e l e d ~ n a n o b o d i e s: ~}$ a new type of targeted probes for imaging antigen expression. Curr Radiopharm. 2008;1:37-41.

23. McGonagle D, Tan AL. What magnetic resonance imaging has told us about the pathogenesis of rheumatoid arthritis: the first 50 years. Arthritis Res Ther. 2008; 10:222.

24. Evans L, Williams AS, Hayes AJ, Jones SA, Nowell M. Suppression of leukocyte infiltration and cartilage degradation by selective inhibition of pre-B cell colonyenhancing factor/visfatin/nicotinamide phosphoribosyltransferase: Apo866-mediated therapy in human fibroblasts and murine collagen-induced arthritis. Arthritis Rheum. 2011;63:1866-1877.

25. Rosenzweig HL, Jann MJ, Vance EE, Planck SR, Rosenbaum JT, Davey MP. Nucleotide-binding oligomerization domain 2 and Toll-like receptor 2 function independently in a murine model of arthritis triggered by intraarticular peptidoglycan. Arthritis Rheum. 2010;62:1051-1059.

26. Chen WT, Mahmood U, Weissleder R, Tung CH. Arthritis imaging using a nearinfrared fluorescence folate-targeted probe. Arthritis Res Ther. 2005;7:R310-R317.

27. Chapman PT, Jamar F, Keelan ETM, Peters AM, Haskard DO. Use of a radiolabeled monoclonal antibody against E-selectin for imaging of endothelial activation in rheumatoid arthritis. Arthritis Rheum. 1996;39:1371-1375.

28. Vogt L, Schmitz N, Kurrer MO, et al. VSIG4, a B7 family-related protein, is a negative regulator of T cell activation. J Clin Invest. 2006;116:2817-2826.

29. Bartok B, Firestein GS. Fibroblast-like synoviocytes: key effector cells in rheumatoid arthritis. Immunol Rev. 2010;233:233-255.

30. Xavier C, Vaneycken I, D'Huyvetter M, et al. Synthesis, preclinical validation, dosimetry, and toxicity of ${ }^{68} \mathrm{Ga}$-NOTA-anti-HER2 Nanobodies for iPET imaging of HER2 receptor expression in cancer. J Nucl Med. 2013;54:776-784.

31. Vegt E, de Jong M, Wetzels JF, et al. Renal toxicity of radiolabeled peptides and antibody fragments: mechanisms, impact on radionuclide therapy, and strategies for prevention. J Nucl Med. 2010;51:1049-1058.

32. Licha K, Welker P, Weinhart M, et al. Fluorescence Imaging with multifunctional polyglycerol sulfates: novel polymeric near-IR probes targeting inflammation. Bioconjug Chem. 2011;22:2453-2460.

33. Hernot $\mathrm{S}$, Unnikrishnan $\mathrm{S}$, Du Z, et al. Nanobody-coupled microbubbles as novel molecular tracer. J Control Release. 2012;158:346-353. 